DOI: https://doi.org/10.46296/gt.v3i6.0013

\title{
CAPACITACIÓN DEL TALENTO HUMANO Y LA GESTIÓN DE LA CALIDAD EN INSTITUCIONES DEL SECTOR SALUD
}

\section{TRAINING OF HUMAN TALENT AND QUALITY MANAGEMENT IN INSTITUTIONS OF THE HEALTH SECTOR}

\author{
Durán-Solórzano Stéfano Alexander ${ }^{1 *}$ \\ Martínez-Minda Héctor Alfredo² \\ ${ }^{1}$ Escuela Superior Politécnica del Litoral, ESPOL. Guayaquil, Ecuador. \\ ${ }^{2}$ Escuela Superior Politécnica del Litoral, ESPOL. Guayaquil, Ecuador.
}

*Correo: sads.2050@gmail.com

\begin{abstract}
Resumen
Este documento está fundamentado en bases teóricas de estudios realizados sobre el desarrollo del talento humano. Las empresas contemporáneas están en una continua actualización de conocimientos impartidos al talento humano, pues las necesidades de adiestramiento y capacitación son una etapa obligada en el escenario laboral, para poder enfrentar el desafío a la apertura de nuevos mercados nacionales e internacionales y de esta manera alcanzar la eficiencia y garantizar la competitividad. Es esencial determinar los canales de capacitación que se han de aplicar, la estructura en la que estará basado; por ejemplo, la comunicación en las diferentes etapas es fundamental, los recursos tecnológicos (TICs) como herramientas básicas para proporcionar información de la empresa a los empleados, en razón de que permitirá hacerlo con efectividad y en menor tiempo, garantizando que las actividades estarán desarrolladas en base a la Gestión de Calidad Total. Se evidencia que las organizaciones deben implementar acciones que desarrollen al personal, de modo que existen muchas maneras de preparación, entre las que sobresalen las capacitaciones virtuales, que ofrecen múltiples ventajas, como: la disponibilidad de horario y accesibilidad, además, la educación virtual fortalece la capacidad del pensamiento crítico.
\end{abstract}

Palabras clave: Formación, gestión, capital humano, empresas.

\begin{abstract}
This document is based on theoretical bases of studies carried out on the development of human talent. Contemporary companies are in a continuous updating of knowledge imparted to human talent, since the needs of training and qualification are an obligatory stage in the labor scenario, in order to face the challenge of opening new national and international markets and thus achieve efficiency and guarantee competitiveness. It is essential to determine the training channels to be applied, the structure on which it will be based; for example, communication at different stages is essential, technological resources (ICTs) as basic tools to provide company information to employees, since it will allow it to be done effectively and in less time, guaranteeing that activities will be carried out based on Total Quality Management. It is evident that organizations must implement actions that develop personnel, so that there are many ways of preparation, among which virtual training stands out, which offer multiple advantages, such as: the availability of hours and accessibility, in addition, virtual education strengthens critical thinking ability.
\end{abstract}

Keywords: Training, management, human capital, companies.

Información del manuscrito:

Fecha de recepción: 19 de marzo de 2020

Fecha de aceptación: 02 de junio de 2020

Fecha de publicación: 10 de julio de 2020 


\section{Introducción}

Actualmente, las empresas contemporáneas están en una continua actualización de conocimientos, impartidos al talento humano, pues las necesidades de adiestramiento y capacitación son una etapa obligada en el escenario laboral, para poder enfrentar el desafío a la apertura de nuevos mercados nacionales e internacionales y de esta manera alcanzar la eficiencia y garantizar la competitividad.

De acuerdo con lo que expresa Mejía et al., (2013), el conocimiento y la capacitación impartida a través de las áreas de talento humano, trasciende hacia una mínima parte del aparato productivo de los negocios, por ende, un elevado porcentaje de profesionales no reciben este beneficio, debido a que no existen los canales de capacitación idóneos de transferencia de conocimiento para todos, pues no se ofrecen cursos, seminarios y talleres de manera frecuente; situación que puede ser originada por omisión o negligencia de la Gestión de los Gerentes de Talento Humano.
Por lo tanto, el objetivo del presente artículo es abordar y discutir la importancia de la gestión del talento humano, respecto a la capacitación y formación y su trascendencia en la gestión de la calidad en empresas contemporáneas.

\section{Gestión del Talento Humano}

La gestión del Talento Humano ha sido incorporada para gestionar procesos, cuyo fin es lograr resultados positivos para la organización, por tal razón es importante comprender su valor, puesto que permite lograr la innovación y un mayor compromiso en su desempeño, lo que contribuye a maximizar la productividad y alcanzar altos niveles de competitividad en las empresas.

Rodríguez (2008, p.9), concluye que: gestionar el Talento Humano se ha convertido en la clave para el éxito empresarial, ya que, sin él, sería prácticamente imposible enfrentar las exigencias actuales $y$ futuras del mercado, gestionarlo es el reto principal que tienen que afrontar, para ello es necesario incorporar una visión más integradora en la mentalidad de los directivos $y$ 
trabajadores de las diferentes Organizaciones y siempre pensar que las propuestas metodológicas que se utilicen deben implementarse en su totalidad para lograr la sinergia funcional del proceso. Una vez logrado esto lo que resta es dirigir el Talento hacia el destino de impacto deseado y con ello se obtendrán los resultados deseados.

En este sentido, el autor destaca la relevancia que tiene la gestión del Talento Humano, para enfrentar un escenario empresarial tan cambiante, donde la tecnología constantemente presenta innovaciones; en tal virtud la estructura organizacional de toda institución debe promover capacitaciones con especialistas, a fin de plantear estrategias metodológicas que provoquen la mejora de los procesos de la organización, para que esta pueda enfrentar los retos que se le presentan y lograr resultados positivos, atendiendo las características de la gestión que promuevan la mejora de los servicios.
Como consecuencia es preciso lograr un equilibrio entre las necesidades de capital humano y la fuerza del trabajo, que facilite el desenvolvimiento para que se pueda cumplir con las expectativas proyectadas. Un factor elemental para el éxito de las organizaciones es la contribución de los conocimientos e ideas innovadoras que con la participación activa basada en la democracia y cooperación, permita el cumplimiento de las metas organizacionales.

\section{Gestión de Procesos Operativos}

La gestión de Procesos (GP), es la herramienta que permite analizar y delimitar las principales actividades, para establecer las mejoras de calidad. Permiten medir de manera periódica los indicadores que informan sobre la situación permanente de dichos procesos.

Las autoridades sanitarias preocupadas por prestar una mejor atención, ya han implementado gestión de procesos en la Atención Primaria y Especializada.

En la actualidad, existe la necesidad de considerar los procesos en el 
área estructural, en razón de permitir

a los especialistas implantar las medidas necesarias, para minimizar las ineficiencias que, repercuten en la salud de los pacientes. Es importante que todos los profesionales direccionen su labor para satisfacer las necesidades y expectativas del paciente con una atención de calidad.

En este sentido, ISO 9000 define proceso como: "Conjunto de actividades mutuamente relacionadas o que interactúan, las cuales transforman elementos de entrada en resultados". En otras palabras, un proceso corresponde a las actividades secuenciadas, cuyo fin es lograr un objetivo.

\subsection{Niveles de procesos}

Los niveles de procesos varían de acuerdo con el tamaño de la organización y podrían ser los siguientes: Alta Dirección, Dirección Intermedia, Mando Intermedio.

Como ya fue aclarado en los párrafos previos, los procesos son las actividades clave requeridas para manejar una organización, por lo que es necesario establecer la jerarquía del proceso. De acuerdo a lo que describe Pérez (2010) se deben considerar los siguientes niveles: nivel macro-proceso y nivel proceso.

El nivel macro-proceso es una representación ya definida de los procesos que desarrolla la organización en su conjunto. Este es el caso del Sistema Nacional de Salud ecuatoriana que presenta una estructura organizacional por procesos, lo cual es positivo por cuanto permite conocer de forma sistemática a la organización, además facilita la interacción entre las diferentes áreas y el trabajo en equipo, lo que conduce a la búsqueda de soluciones de los problemas comunes que suelen presentarse en la organización.

Por su parte, en los establecimientos de salud el nivel proceso corresponde a un proceso de atención concreta, es decir que se representa la forma en que se deben hacer las actividades y delegar responsabilidades. Este nivel está basado en la identificación, análisis, definición, monitoreo-evaluación de los procesos de la organización.

\subsection{Determinación de procesos}

Todo proceso se encuentra al alcance del Sistema de Gestión de Calidad y tiene como fin brindar al 
cliente/usuario un servicio correcto que satisfaga sus expectativas, con el mejor rendimiento tanto en costo, servicio y calidad. Por tal razón el procedimiento es la forma concreta de llevar a término un proceso, los resultados dependen de la capacidad y conocimientos de las personas involucradas, así también como de los recursos con que cuenta la organización.

Para determinar los procesos es necesario considerar varios criterios, en este caso se lo realizará en referencia a procesos estratégicos, claves y de apoyo. En el caso de los servicios de salud, los procesos claves son los que están inmersos directamente a la atención médica del paciente, por ejemplo, se puede citar el proceso a seguir en el área de la unidad de emergencia.

En el presente artículo se consideró la estructura Organizacional por Procesos de la Dirección Provincial de Salud, misma que sirvió para facilitar la comprensión integral y la localización de puntos que requieren mejora.

MINISTERIO DE SALUD PÚBLICA

ESTRUCTURA ORGANIZACIONAL POR PROCESOS-DIRECCIÓN PROVINCIAL DE SALUD

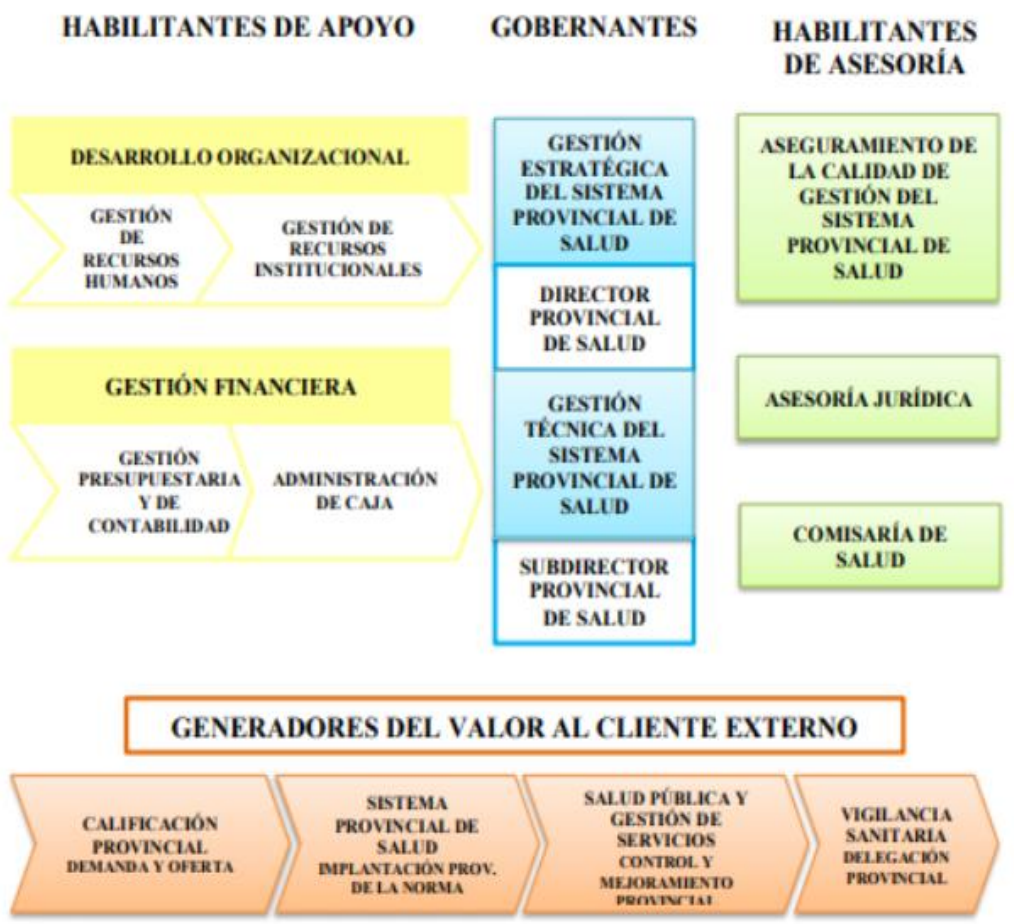

Figura 1. Estructura organizacional por procesos. Ministerio de Salud Pública (2011:52) 
Paganini \& Arrondo (2011:4), consideran importante seis pasos para la documentación, análisis y mejora de los procesos críticos en un centro de salud.

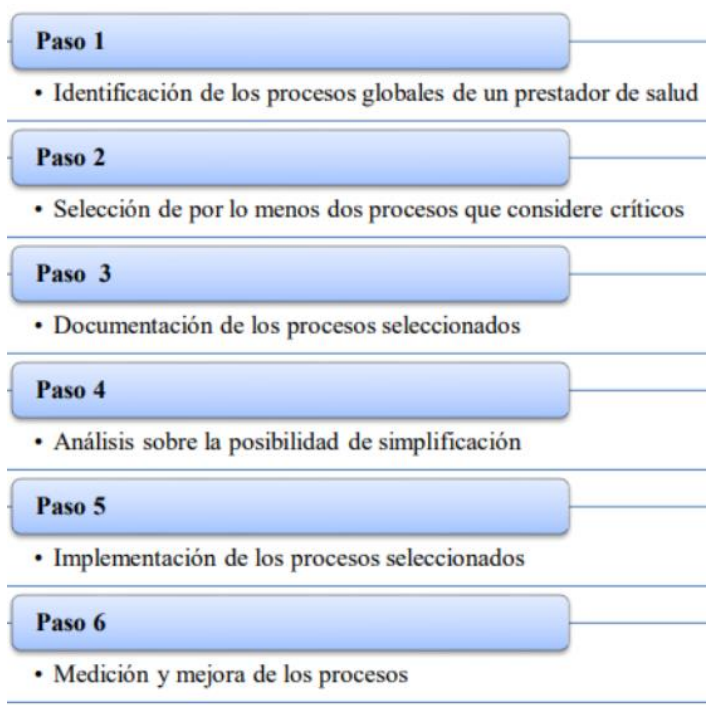

Figura 2. Análisis y mejora de los procesos críticos. Fuente: Paganini \& Arrondo (2011)

\section{Identificación de los procesos de} un prestador de salud. Identifica los procesos que realiza cotidianamente un prestador de salud, mediante el diagrama de un mapa que debe ser adaptado de acuerdo al departamento o a la actividad que realiza, cuando se realiza el control de infecciones, por ejemplo, se pueden identificar varias actividades que contribuyen al logro del objetivo propuesto, estas pueden iniciar por el control que efectúa el comité de infecciones, el infectólogo, área de enfermería, mucamas, entre otros prestadores. Como se puede observar, un proceso generalmente no comienza y termina en un sector, sino que cruza por diferentes áreas.

Selección de procesos considerados críticos. Luego de identificar los procesos, se debe prestar atención a los puntos críticos, con el fin de mejorar la calidad de atención. Cabe mencionar que estos conllevan riesgos en diferentes ámbitos por lo que deben ser monitoreados. Entre los procesos críticos se pueden mencionar: procesos de internación, cirugía, guardia, seguridad del paciente y su familia, historias clínicas, insumos, información, recursos humanos, auditoría interna.

\section{Documentación de los procesos} seleccionados. Identificados los procesos críticos, es necesario investigar cómo se realiza cada uno de ellos, para proceder a documentarlos. La información puede ser obtenida a través de entrevistas abiertas, observación o mediante la descripción del proceso del cual participa la persona.

Análisis sobre la posibilidad de simplificación. La información documentada facilita la revisión y 
esto a su vez permite identificar actividades que podrían ser mejoradas para lograr la eficacia y calidad.

\section{Ejecución de los procesos} seleccionados. Una vez seleccionados los procesos, deben ser socializados para luego difundirlos, la capacitación y entrenamiento del personal tiene relevante importancia, para su correcta ejecución.

Medición y mejora de los procesos. La gestión por procesos tiene como fin la mejora continua. Por tal razón las mejoras deben ser orientadas a lograr la perfección el proceso, disminuir costos, tiempo e incrementar la capacidad, seguridad y satisfacción del paciente y su familia. Para realizar este análisis, se sugiere apoyarse en herramientas gráficas, mapas de procesos, capacitaciones virtuales que permitan lograr el éxito.

\section{Análisis de los canales de capacitación}

La aplicación de un plan integral de capacitación, debidamente estructurado y constituido con temáticas necesarias para el personal, surge como alternativa para fortalecer las capacidades de la atención al usuario y de esta manera superar los diferentes inconvenientes o problemas que suelen presentarse durante el cumplimiento de las actividades laborales.

Es esencial determinar los canales de capacitación que se han de aplicar, la estructura en la que estará basado; por ejemplo, la comunicación en las diferentes etapas es fundamental, los recursos tecnológicos (TICs) como herramientas básicas para proporcionar información de la empresa a los empleados, en razón de que permitirá hacerlo con efectividad y en menor tiempo, las actividades estarán desarrolladas en base a la Gestión de Calidad Total.

Los programas de capacitación pueden desarrollarse a través de dos modalidades básicas, semipresencial y a distancia, bajo el control, seguimiento y evaluación, de conocimiento, de nuevos enfoques de atención y servicios de salud. Las clases podrán ser dictadas por funcionarios del hospital, conferencistas especializados, videos, este tipo de capacitaciones favorecen el trabajo en equipo, 
direccionado por tutores y el asistente tecnológico.

\subsection{Trascendencia de la} capacitación del Talento Humano

Actualmente, la capacitación del talento humano es la afirmación a la necesidad de las instituciones 0 empresas de contar con una mano de obra calificada y productiva. La obsolescencia, así mismo es uno de los argumentos por el cual las instituciones se esmeran por capacitar a su personal, pues de esta forma se actualizan sus discernimientos con los nuevos métodos y técnicas de trabajo que avalan eficacia.

Chiavenato (2007, p.390) expresa que:

La detección de las necesidades es la primera etapa de la capacitación y se refiere al diagnóstico preliminar que se precisa hacer. La detección de las necesidades de capacitación se puede efectuar considerando tres niveles de análisis: 1) Nivel de análisis de toda la organización: en el sistema organizacional, 2) Nivel de análisis de los recursos humanos: el sistema de capacitación y 3) Nivel de análisis de las operaciones y tareas.

Para las organizaciones y empresas, la capacitación del talento humano debe ser trascendental, debido a que contribuye al adelanto personal y profesional de los sujetos, a la vez que origina beneficios significativos para la empresa, por tal razón la adquisición de nuevos conocimientos adquiere una fuerte importancia, en virtud de que el personal es más competitivo dentro de la organización, porque los pone en práctica en cada uno de los procedimientos establecidos.

Sin duda alguna la capacitación, permite el desarrollo de habilidades y creatividad del capital humano, por este motivo se deben gestionar adecuadamente los respectivos entrenamientos.

\subsection{Ventajas de la capacitación}

La capacitación a todos los grados compone una de las principales inversiones en talento humano y una de las primordiales fuentes de satisfacción para el empleado y la organización. 
Según León (2010, p.24): La capacitación a las organizaciones ofrece las siguientes ventajas:

- Incrementa el conocimiento del puesto a todos los horizontes.

- Empuja a más rentabilidad y a actitudes más efectivas.

- Establece mejor imagen.

- Se origina la comunicación a todo el organismo.

- Mejora la conexión jefessubordinados.

- Se aligera la toma de decisiones y la salida de problemas.

- Disminuye la tensión y admite el manejo de áreas de problemas.

- Ayuda a la formación de líderes y directores.

- Origina el adelanto con vistas a la promoción.

Por su parte, los beneficios de la capacitación al personal son:

- Contribuye efectivamente en el manejo de aprietos y tensiones.

- Fomenta la confianza, la posición y el desarrollo.

- Remonta el nivel de complacencia con el puesto.

- Forja líderes y perfecciona las aptitudes expresivas.
- Aparta los temores a la incapacidad o la ignorancia propia.

- Accede al logro de metas especiales.

- Despliega un sentido de adelanto en numerosos campos.

5. Las TIC como recursos tecnológicos de capacitación

En la actualidad, el uso de la tecnología es casi imprescindible en todos los campos de la ciencia, pues en múltiples actividades se constituyen en recursos que muchos profesionales utilizan en razón de que facilita el trabajo y rompe la barrera del tiempo. Desde la aparición del internet se han realizado cambios vertiginosos, en el ámbito educativo, comercial, social y de la salud, muchas empresas contemporáneas utilizan la tecnología para alcanzar sus objetivos.

Introducir las nuevas tecnologías en el ámbito empresarial es un reto, en razón de que es un proceso de grandes cambios, lo cual requiere de responsabilidad y dedicación. Pero, sin duda alguna, la aplicación de las Tecnologías de la Información y la Comunicación en las organizaciones aporta significativamente en el 
control de las actividades y ayuda a la toma decisiones, por lo que actualmente, se ha convertido en un componente central de las empresas que pretenden mantener su competitividad en el mercado.

Las tecnologías informáticas se desarrollan de diferentes maneras, una más sistemática que otras; por lo que implican propuestas de capacitación, con costos muy reducidos. Las TIC involucran aprendizajes, pues al ofrecer una amplia plataforma cargada de información, constituyen una estrategia de capacitación que se apoya en un conjunto de saberes para afrontar los procesos de enseñanza.

Las TIC pueden ser consideradas como una herramienta a la hora de diseñar planes de capacitación para diferentes ámbitos laborales, cuyo impacto es mayor en países en desarrollo, y los efectos han sido positivos sobre los niveles de productividad de las empresas; cabe mencionar que además de mejorar la productividad laboral, tienen incidencia directamente sobre el crecimiento económico de un país.

Se pueden contemplar efectos positivos sobre la inversión, el capital humano, el capital social, el comercio y salud.

El Ministerio de Telecomunicaciones y de la Sociedad de la Información, (2014:10) sostiene que: fomentar el desarrollo de una industria de la TIC, competente y generadora de empleos para la creación de productos y servicios tecnológicos, permite incrementar su contribución socioeconómica del país que impacta directamente sobre el desarrollo. En tal sentido, hay al menos tres conceptos implícitos en el modo en que construyen su vínculo que resulta necesario atender: la idea de conocimiento, de práctica y de distancia respecto de la tecnología, en todos estos casos los usos fructíferos y fluidos de las TIC requieren conocimientos previos.

La capacitación a través de las plataformas virtuales propicia situaciones que optimizan las actividades en la organización y favorece la producción. Un aspecto importante en este sentido es que se dispone de mejor manera de las tecnologías, ya que están al alcance de todos, lo que contempla una multiplicidad de usos posibles, por lo que es un recurso para la construcción del conocimiento, a 
través de los capacitadores mediante el diseño de una planificación institucional.

\subsection{Estrategias de capacitación basadas en TIC}

Diversos estudios realizados en diferentes países demuestran que la implementación de las TIC es fundamental para generar mejoras y ser competitivos. No cabe duda que el desarrollo tecnológico contribuye a que una población consiga superar la brecha digital que imponen las TIC entre distintas organizaciones.

Leibowicz (2011, p.5) menciona que:

Para que se apliquen las TIC de una manera efectiva que se refleje en un aumento de la productividad e innovación, es preciso que las empresas acompañen la incorporación de las tecnologías con definición de estrategias, de políticas de gestión y sistemas de organización del trabajo orientados a hacer visibles procesos de comunicación y aprendizaje.

Desde esta perspectiva se podría decir que las empresas que implementen las TIC, fortalecerán su base empresarial, en el desarrollo de conocimientos y capacidades de su capital humano. Previo al análisis de diferentes factores que inciden en su aplicación, debido a que en las organizaciones intervienen factores tecnológicos y culturales que inciden en mayor o menor medida en la incorporación de las TIC. Entre los factores tecnológicos se encuentran los relativos a infraestructura, hardware, software, Internet y a programas informáticos. Los factores culturales hacen referencia a la alfabetización informática, o capacidad de utilizar las TIC para el desarrollo organizacional de aprendizaje e innovación.

\section{Empresas contemporáneas}

La sociedad actual está caracterizada por el auge del conocimiento científico-tecnológico, la globalización impone retos para los empresarios que pretenden lograr la pertinencia de sus empresas, ante esta situación surge la necesidad de realizar diseños de estrategias en relación con la proyección de sus intereses y es aquí donde radica la importancia de invertir en el desarrollo del talento humano, que garantice la flexibilidad y rentabilidad de la organización. 
En este sentido, Vasquez (2010) refleja que:

"El logro de la satisfacción de los clientes a través del establecimiento adecuado de todos sus requisitos y el cumplimiento de los mismos con procesos eficientes, que permita así a la organización ser competitiva en la industria y beneficie al cliente con precios razonables."

Las empresas $\mathrm{u}$ organizaciones perduran y mantienen el éxito, porque innovan teórica $y$ conceptualmente para lograr un desempeño de calidad, y de esta manera genera la confianza necesaria en los negocios y cultiva un auténtico sentido del trabajo cooperativo que permite cimentar y edificar su prosperidad a nivel social.

\subsection{Gestión de la Calidad}

A mediados del siglo XIX surge la necesidad de utilizar normas de calidad para las empresas $u$ organizaciones comerciales 0 de servicio, pues la producción aumenta de manera acelerada. Aunque en el territorio ecuatoriano la industrialización fue tardía, no obstante, el mismo capitalismo absorbió a los pequeños países bajo las condiciones de producción estándar.

Las Normas ISO (Organización Internacional de Normalización) pertenecen a la federación mundial de organismos nacionales de normalización (organismos miembros de ISO). El trabajo de preparación de las Normas Internacionales, normalmente se realiza a través de los comités técnicos de ISO. Cada organismo miembro interesado en una materia para la cual se haya establecido un comité técnico, tiene el derecho de estar representado en dicho comité. Las organizaciones internacionales, públicas y privadas, en coordinación con ISO, también participan y colaboran estrechamente con la Comisión Electrotécnica Internacional (IEC) en todas las materias de normalización electrotécnica.

Las normas ISO 9001 (2008):

Son normas de sistema de gestión de la calidad que se han diseñado para complementarse entre sí, pero también pueden utilizarse de manera independiente. Están 
centradas en la eficacia del sistema de gestión de la calidad para satisfacer los requisitos del cliente. La certificación de esta norma permite alcanzar el cumplimiento de las exigencias de los clientes. Hoy en día para las empresas es una estrategia que le permite reconocimiento en el mercado, por lo que se ha vuelto la columna vertebral de las organizaciones.

Las normas de la serie ISO son aplicables tanto en un marco nacional como internacional. Las empresas, instituciones y organizaciones nacionales saben que la aplicación de estas normas es el camino para abrir oportunidades en nuevos mercados y mejorar su competitividad y de esta manera hacer frente a la globalización.

\section{Conclusiones}

A partir de la revisión realizada se concluye que una gestión por procesos, basados en las Normas ISO 9001:2008, ofrece múltiples ventajas, en razón de permitir la formalización de estrategias organizacionales que facilitan los procedimientos, control de registros, designación de funciones y mejora continua, en las diferentes áreas de una institución del sector salud.

Es evidente que las organizaciones deben implementar acciones que desarrollen al personal y no necesariamente se pueden realizar a través de cursos directos, porque existen muchas maneras de preparación, entre las que sobresalen en los actuales momentos, se encuentran las capacitaciones virtuales, que ofrecen grandes ventajas, como: la disponibilidad de horario y accesibilidad, además, la educación virtual fortalece la capacidad del pensamiento crítico.

\section{Bibliografía}

Chiavenato, I. (2007). "Gestión Del Talento Humano", Octava edición. México: McGrawhill.

ISO. (2008). Norma ISO 9001:2008. Norma Internacional.

Ginebra-Suiza:

Cuarta edición.

Leibowicz, J. (2011). Estrategias de Capacitación Basadas en TIC para Mipyme. p. 5. CINTERFOR. 
León, R. Y. (2010). Importancia de la Capacitación del Personal en las Utilidades de la Empresa. Disponible

en: http://www.gerenciayempresa .com/documentos/articulos/IM PORTANCIA_DE_LA_CAPA CITACION.pdf

Mejía-Giraldo, A., Bravo-Castillo, M., \& Montoya-Serrano, A. (2013). El factor del talento humano en las organizaciones. Ingeniería industrial, 34(1), 2-11.

Ministerio de Salud Pública del Ecuador. (2008). Sumario: Función Ejecutiva. Acuerdo: Ministerio de Salud Pública: Quito. Registro Oficial № 151.

Ministerio de Telecomunicaciones y de la Sociedad de la Información. (2014). Tecnologías de la información y comunicación para el desarrollo. Disponible en: http://www.industrias.ec/archi vos/CIG/file/CARTELERA/MI NTELTIC\%20para\%20el\%20 Desarrollo.pd

Paganini \& Arrondo. (2011). Gestión por Procesos. La Plata España: Universidad Nacional de La Plata.
Pérez, J. A. (2010). Gestión por procesos. Madrid - España: ESIC Editorial.

Rodríguez, C. Y. (2008). La Gestión del Talento Humano como fuente generadora de innovación tecnológica. Propuesta Metodológica para su aplicación. Recuperado el 19 de 11 de 2014, de La Gestión del Talento Humano como fuente generadora de innovación tecnológica. Propuesta Metodológica para su aplicación. Disponible en: innohttp://www.bibliociencias. cu/gsdl/collect/eventos/import /Gestion_talento_humano.pdf Vasquez, M. (2010). La calidad organizacional sostenible, en su inicio, es cuestión de actitud positiva y liderazgo gerencial". Disponible en: http://www.degerencia.com/ar ticulo/lideres_o_gerentes_par a_la_busqueda_eficaz 